\title{
Some Curvature Conditions on a Para-Sasakian Manifold with Canonical Paracontact Connection
}

\author{
Bilal Eftal Acet, ${ }^{1}$ Erol Kılıç, ${ }^{2}$ and Selcen Yüksel Perktaş ${ }^{1}$ \\ ${ }^{1}$ Department of Mathematics, Arts and Science Faculty, Adryaman University, 02040 Adryaman, Turkey \\ ${ }^{2}$ Department of Mathematics, Arts and Science Faculty, Inönü University, 44280 Malatya, Turkey
}

Correspondence should be addressed to Selcen Yüksel Perktaş, sperktas@adiyaman.edu.tr

Received 2 October 2012; Accepted 2 December 2012

Academic Editor: Jerzy Dydak

Copyright ( 2012 Bilal Eftal Acet et al. This is an open access article distributed under the Creative Commons Attribution License, which permits unrestricted use, distribution, and reproduction in any medium, provided the original work is properly cited.

We study canonical paracontact connection on a para-Sasakian manifold. We prove that a Ricci-flat para-Sasakian manifold with respect to canonical paracontact connection is an $\eta$-Einstein manifold. We also investigate some properties of curvature tensor, conformal curvature tensor, $W_{2^{-}}$ curvature tensor, concircular curvature tensor, projective curvature tensor, and pseudo-projective curvature tensor with respect to canonical paracontact connection on a para-Sasakian manifold. It is shown that a concircularly flat para-Sasakian manifold with respect to canonical paracontact connection is of constant scalar curvature. We give some characterizations for pseudo-projectively flat para-Sasakian manifolds.

\section{Introduction}

In 1976, Sato [1] introduced the almost paracontact structure $(\varphi, \xi, \eta)$ satisfying $\varphi^{2}=I-\eta \otimes \xi$ and $\eta(\xi)=1$ on a differentiable manifold. Although the structure is an analogue of the almost contact structure [2,3], it is closely related to almost product structure (in contrast to almost contact structure, which is related to almost complex structure). It is well known that an almost contact manifold is always odd-dimensional but an almost paracontact manifold defined by Sato [1] could be even dimensional as well. Takahashi [4] defined almost contact manifolds equipped with an associated pseudo-Riemannian metric. In particular he studied Sasakian manifolds equipped with an associated pseudo-Riemannian metric. Also, in 1989, Matsumoto [5] replaced the structure vector field $\xi$ by $-\xi$ in an almost paracontact manifold and associated a Lorentzian metric with the resulting structure and called it a Lorentzian almost paracontact structure. It is obvious that in a Lorentzian almost paracontact manifold, the pseudo-Riemannian metric has only signature 1 and the structure vector field $\xi$ is always 
timelike. These circumstances motivated the authors [6] to associate a pseudo-Riemannian metric, not necessarily Lorentzian, with an almost paracontact structure.

Kaneyuki and Konzai [7] defined the almost paracontact structure on pseudoRiemannian manifold $M$ of dimension $(2 n+1)$ and constructed the almost paracomplex structure on $M^{2 n+1} \times \mathbb{R}$. Zamkovoy [8] associated the almost paracontact structure introduced in [7] to a pseudo-Riemannian metric of signature $(n+1, n)$ and showed that any almost paracontact structure admits such a pseudo-Riemannian metric which is called compatible metric.

Tanaka-Webster connection has been introduced by Tanno [9] as a generalization of the well-known connection defined by Tanaka [10] and, independently, by Webster [11], in context of CR geometry. In a paracontact metric manifold Zamkovoy [8] introduced a canonical connection which plays the same role of the (generalized) Tanaka-Webster connection [9] in paracontact geometry. In this study we define a canonical paracontact connection on a para-Sasakian manifold which seems to be the paracontact analogue of the (generalized) Tanaka-Webster connection.

In the present paper we study canonical paracontact connection on a para-Sasakian manifold. Section 2 is devoted to preliminaries. In Section 3, we investigate the relation between curvature tensor (resp., Ricci tensor) with respect to canonical paracontact connection and curvature tensor (resp., Ricci tensor) with respect to Levi-Civita connection. In Section 4, conformal curvature tensor of a para-Sasakian manifold with respect to canonical paracontact connection is obtained. Section 5 contains the expression of $\bar{W}_{2}$-curvature tensor. In Section 6, we study a para-Sasakian manifold satisfying the condition $\bar{C}(\xi, X) \cdot \bar{R}=0$, where $\bar{C}(\xi, X)$ is considered as a derivation of the tensor algebra at each point of the manifold, $\bar{R}$ is the curvature tensor, and $\bar{C}$ is the conformal curvature tensor with respect to canonical paracontact connection. In Section 7, we obtain some equations in terms of Ricci tensor on a para-Sasakian manifold satisfying $\bar{W}_{2}(\xi, X) \cdot \bar{R}=0$ and $\bar{W}_{2}(\xi, X) \cdot \bar{W}_{2}=0$, respectively. In Section 8 it is proved that a concircularly flat para-Sasakian manifold is of constant scalar curvature. Section 9 is devoted to pseudo-projectively flat para-Sasakian manifolds. In Section 10, we show that a para-Sasakian manifold satisfying $\bar{Z}(\xi, X) \cdot \bar{R}=0$ with respect to canonical paracontact connection, is either of constant scalar curvature or an $\eta$-Einstein manifold. Also, it is proved that if the condition $\bar{Z}(\xi, X) \cdot \bar{S}=0$ holds on a paraSasakian manifold with respect to canonical paracontact connection, then the scalar curvature is constant. In Section 11, we give some characterizations for para-Sasakian manifolds with canonical paracontact connection satisfying $\bar{P} \bar{P}(\xi, X) \cdot \bar{R}=0$ and $\bar{P} \bar{P}(\xi, X) \cdot \bar{S}=0$, respectively. In the last section it is shown that a para-Sasakian manifold on which the condition $\bar{P}(\xi, X)$. $\bar{P} \bar{P}=0$ holds, is either of constant scalar curvature or an $\eta$-Einstein manifold.

\section{Preliminaries}

A differentiable manifold $M$ of dimension $(2 n+1)$ is called almost paracontact manifold with the almost paracontact structure $(\varphi, \xi, \eta)$ if it admits a tensor field $\varphi$ of type $(1,1)$, a vector field $\xi$, a 1-form $\eta$ satisfying the following conditions [7]:

$$
\begin{gathered}
\varphi^{2}=I-\eta \otimes \xi, \\
\eta(\xi)=1, \quad \varphi \xi=0,
\end{gathered}
$$


where $I$ denotes the identity transformation. Moreover, the tensor field $\varphi$ induces an almost paracomplex structure on the paracontact distribution $D=\operatorname{ker} \eta$; that is, the eigendistributions $D^{ \pm}$corresponding to the eigenvalues \pm 1 of $\varphi$ are both $n$-dimensional. As an immediate consequence of the conditions (2.2) we have

$$
\eta \circ \varphi=0, \quad \operatorname{rank}(\varphi)=2 n
$$

If a $(2 n+1)$-dimensional almost paracontact manifold $M$ with an almost paracontact structure $(\varphi, \xi, \eta)$ admits a pseudo-Riemannian metric $g$ such that [8]

$$
g(\varphi X, \varphi Y)=-g(X, Y)+\eta(X) \eta(Y), \quad X, Y \in \Gamma(T M)
$$

then we say that $M$ is an almost paracontact metric manifold with an almost paracontact metric structure $(\varphi, \xi, \eta, g)$, and such metric $g$ is called compatible metric. Any compatible metric $g$ is necessarily of signature $(n+1, n)$.

From (2.4) it can be easily seen that [8]

$$
\begin{gathered}
g(X, \varphi Y)=-g(\varphi X, Y), \\
g(X, \xi)=\eta(X),
\end{gathered}
$$

for any $X, Y \in \Gamma(T M)$. The fundamental 2-form of $M$ is defined by

$$
\Phi(X, Y)=g(X, \varphi Y)
$$

An almost paracontact metric structure becomes a paracontact metric structure if $g(X, \varphi Y)=$ $d \eta(X, Y)$, for all vector fields $X, Y \in \Gamma(T M)$, where $d \eta(X, Y)=(1 / 2)\{X \eta(Y)-Y \eta(X)-$ $\eta([X, Y])\}$.

For a $(2 n+1)$-dimensional manifold $M$ with an almost paracontact metric structure $(\varphi, \xi, \eta, g)$ one can also construct a local orthonormal basis. Let $U$ be coordinate neighborhood on $M$ and $X_{1}$ any unit vector field on $U$ orthogonal to $\xi$. Then $\varphi X_{1}$ is a vector field orthogonal to both $X_{1}$ and $\xi$, and $\left|\varphi X_{1}\right|^{2}=-1$. Now choose a unit vector field $X_{2}$ orthogonal to $\xi, X_{1}$ and $\varphi X_{1}$. Then $\varphi X_{2}$ is also a vector field orthogonal to $\xi, X_{1}, \varphi X_{1}$, and $X_{2}$ and $\left|\varphi X_{2}\right|^{2}=-1$. Proceeding in this way we obtain a local orthonormal basis $\left(X_{i}, \varphi X_{i}, \xi\right),(i=1,2, \ldots, n)$ called a $\varphi$-basis [8].

Remark 2.1. It is also known that a differentiable manifold has an almost paracontact metric structure if it admits a Riemannian metric $g$ such that $g(\varphi X, \varphi Y)=g(X, Y)-\eta(X) \eta(Y)$ (see [1]). But in our paper the metric $g$ is pseudo-Riemannian and satisfies condition (2.4).

Recall that an almost paracomplex structure [12] on a $2 n$-dimensional manifold is a tensor field $J$ of type $(1,1)$ such that $J^{2}=I$ and eigensubbundles $T^{+}, T^{-}$corresponding to the eigenvalue $1,-1$ of $J$, respectively, have equal dimensional $n$. The Nijenhuis tensor $N$ of $J$, given by

$$
N_{J}(X, Y)=[J X, J Y]+[X, Y]-J[J X, Y]-J[X, J Y], \quad X, Y \in \Gamma(T M),
$$


is the obstruction for the integrability of the eigensubbundles $T^{+}, T^{-}$. If $N=0$, then the almost paracomplex structure is called paracomplex or integrable [13].

Let $M^{2 n+1}$ be an almost paracontact metric manifold with structure $(\varphi, \xi, \eta)$ and consider the manifold $M^{2 n+1} \times \mathbb{R}$. We denote a vector field on $M^{2 n+1} \times \mathbb{R}$, by $(X, f(d / d t))$ where $X$ is tangent to $M^{2 n+1}, t$ is the coordinate on $\mathbb{R}$, and $f$ is a differentiable function on $M^{2 n+1} \times \mathbb{R}$. An almost paracomplex structure $J$ on $M^{2 n+1} \times \mathbb{R}$ is defined in [14] by

$$
J\left(X, f \frac{d}{d t}\right)=\left(\varphi X+f \xi, \eta(X) \frac{d}{d t}\right)
$$

If $J$ is integrable we say that the almost paracontact structure $(\varphi, \xi, \eta)$ is normal.

A normal paracontact metric manifold is a para-Sasakian manifold. An almost paracontact metric structure $(\varphi, \xi, \eta, g)$ on a $M^{2 n+1}$ is para-Sasakian manifold if and only if [8]

$$
\left(\nabla_{X} \varphi\right) Y=-g(X, Y) \xi+\eta(Y) X
$$

where $X, Y \in \Gamma(T M)$ and $\nabla$ is Levi-Civita connection of $M$.

From (2.10), it can be seen that

$$
\nabla_{X} \xi=-\varphi X
$$

Also in a para-Sasakian manifold, the following relations hold [8]:

$$
\begin{gathered}
g(R(X, Y) Z, \xi)=\eta(R(X, Y) Z)=g(X, Z) \eta(Y)-g(Y, Z) \eta(X) \\
R(X, Y) \xi=\eta(X) Y-\eta(Y) X \\
R(\xi, X) Y=-g(X, Y) \xi+\eta(Y) X \\
R(\xi, X) \xi=X-\eta(X) \xi \\
S(X, \xi)=-2 n \eta(X)
\end{gathered}
$$

for any vector fields $X, Y, Z \in \Gamma(T M)$. Here, $R$ is Riemannian curvature tensor and $S$ is Ricci tensor defined by $S(X, Y)=g(Q X, Y)$, where $Q$ is Ricci operator.

In the following we consider the connection $\bar{\nabla}$ defined by [9]

$$
\bar{\nabla}_{X} Y=\nabla_{X} Y+\eta(X) \varphi Y-\eta(Y) \nabla_{X} \xi+\left(\left(\nabla_{X} \eta\right) Y\right) \xi
$$

where $X, Y \in \Gamma(T M)$. If we use (2.11) in (2.17), then we obtain 
International Journal of Mathematics and Mathematical Sciences

$$
\bar{\nabla}_{X} Y=\nabla_{X} Y+\eta(X) \varphi Y+\eta(Y) \varphi X+g(X, \varphi Y) \xi .
$$

Definition 2.2. We call the connection $\bar{\nabla}$ defined by (2.18) on a para-Sasakian manifold the canonical paracontact connection on a para-Sasakian manifold.

Proposition 2.3. On a para-Sasakian manifold the connection $\bar{\nabla}$ has the following properties:

$$
\begin{gathered}
\bar{\nabla} \eta=0, \quad \bar{\nabla} g=0, \quad \bar{\nabla} \xi=0, \\
\left(\bar{\nabla}_{X} \varphi\right) Y=\left(\nabla_{X} \varphi\right) Y+g(X, Y) \xi-\eta(Y) X
\end{gathered}
$$

for all $X, Y \in \Gamma(T M)$.

Proof. Calculation is straightforward by using (2.18).

Furthermore, we define homeomorphisms $\bar{C}(X, Y) \cdot \bar{R}=0, \bar{W}_{2}(X, Y) \cdot \bar{R}=0, \bar{W}_{2}(X, Y)$. $\bar{W}_{2}=0, \bar{Z}(X, Y) \cdot \bar{R}=0, \bar{P} \bar{P}(X, Y) \cdot \bar{R}=0$ and $\bar{P}(X, Y) \cdot \bar{P} \bar{P}=0$ as follows:

$$
\begin{aligned}
(\bar{C}(X, Y) \cdot \bar{R})(U, V) W= & \bar{C}(X, Y) \bar{R}(U, V) W-\bar{R}(\bar{C}(X, Y) U, V) W \\
& -\bar{R}(U, \bar{C}(X, Y) V) W-\bar{R}(U, V) \bar{C}(X, Y) W \\
= & 0, \\
\left(\bar{W}_{2}(X, Y) \cdot \bar{R}\right)(U, V) W= & \bar{W}_{2}(X, Y) \bar{R}(U, V) W \\
& -\bar{R}\left(\bar{W}_{2}(X, Y) U, V\right) W-\bar{R}\left(U, \bar{W}_{2}(X, Y) V\right) W \\
& -\bar{R}(U, V) \bar{W}_{2}(X, Y) W \\
= & 0, \\
\left(\bar{W}_{2}(X, Y) \cdot \bar{W}_{2}\right)(U, V) W= & \bar{W}_{2}(X, Y) \bar{W}_{2}(U, V) W \\
& -\bar{W}_{2}\left(\bar{W}_{2}(X, Y) U, V\right) W-\bar{W}_{2}\left(U, \bar{W}_{2}(X, Y) V\right) W \\
& -\bar{W}_{2}(U, V) \bar{W}_{2}(X, Y) W \\
= & 0,
\end{aligned}
$$




$$
\begin{aligned}
(\bar{Z}(X, Y) \cdot \bar{R})(U, V) W= & \bar{Z}(X, Y) \bar{R}(U, V) W-\bar{R}(\bar{Z}(X, Y) U, V) W \\
& -\bar{R}(U, \bar{Z}(X, Y) V) W-\bar{R}(U, V) \bar{Z}(X, Y) W \\
= & 0, \\
(\bar{P} \bar{P}(X, Y) \cdot \bar{R})(U, V) W= & \bar{P} \bar{P}(X, Y) \bar{R}(U, V) W-\bar{R}(\bar{P} \bar{P}(X, Y) U, V) W \\
& -\bar{R}(U, \bar{P} \bar{P}(X, Y) V) W-\bar{R}(U, V) \bar{P} \bar{P}(X, Y) W \\
= & 0, \quad \\
(\bar{P}(X, Y) \cdot \bar{P} \bar{P})(U, V) W= & \bar{P}(X, Y) \bar{P} \bar{P}(U, V) W-\bar{P} \bar{P}(\bar{P}(X, Y) U, V) W \\
& -\bar{P} \bar{P}(U, \bar{P}(X, Y) V) W-\bar{P} \bar{P}(U, V) \bar{P}(X, Y) W \\
= & 0, \quad
\end{aligned}
$$

for all $X, Y, U, V, W \in \Gamma(T M)$.

\section{Curvature Tensor}

Let $M^{2 n+1}$ be a para-Sasakian manifold. The curvature tensor $\bar{R}$ of $M$ with respect to the canonical paracontact connection $\bar{\nabla}$ is defined by

$$
\bar{R}(X, Y) Z=\bar{\nabla}_{X} \bar{\nabla}_{Y} Z-\bar{\nabla}_{Y} \bar{\nabla}_{X} Z-\bar{\nabla}_{[X, Y]} Z,
$$

for any $X, Y, Z \in \Gamma(T M)$.

By using (2.18) in (3.1) we obtain

$$
\begin{aligned}
\bar{R}(X, Y) Z= & R(X, Y) Z+g(Y, Z) \eta(X) \xi-g(X, Z) \eta(Y) \xi \\
& +\eta(Y) \eta(Z) X-\eta(X) \eta(Z) Y+2 g(X, \varphi Y) \varphi Z \\
& +g(X, \varphi Z) \varphi Y-g(Y, \varphi Z) \varphi X
\end{aligned}
$$

where $R(X, Y) Z=\nabla_{X} \nabla_{Y} Z-\nabla_{Y} \nabla_{X} Z-\nabla_{[X, Y]} Z$ is curvature tensor of $M$ with respect to LeviCivita connection $\nabla$.

Let $T$ and $\bar{T}$ be curvature tensors of type $(0,4)$ with respect to Levi-Civita connection $\nabla$ and the canonical paracontact connection $\bar{\nabla}$, respectively, given by

$$
\begin{aligned}
& T(X, Y, Z, W)=g(R(X, Y) Z, W) \\
& \bar{T}(X, Y, Z, W)=g(\bar{R}(X, Y) Z, W)
\end{aligned}
$$

for all $X, Y, Z, W \in \Gamma(T M)$. 
International Journal of Mathematics and Mathematical Sciences

Proposition 3.1. In a para-Sasakian manifold one has,

$$
\begin{gathered}
\bar{R}(X, Y) Z+\bar{R}(Y, Z) X+\bar{R}(Z, X) Y=0 \\
\bar{T}(X, Y, Z, W)+\bar{T}(Y, X, Z, W)=0 \\
\bar{T}(X, Y, Z, W)+\bar{T}(X, Y, W, Z)=0 \\
\bar{T}(X, Y, Z, W)-\bar{T}(Z, W, X, Y)=0
\end{gathered}
$$

where $X, Y, Z, W \in \Gamma(T M)$.

Proof. Using (3.2) and first Bianchi identity with respect to Levi-Civita connection $\nabla$ we obtain

$$
\begin{aligned}
& \bar{R}(X, Y) Z+\bar{R}(Y, Z) X+\bar{R}(Z, X) Y \\
&= 2 g(X, \varphi Y) \varphi Z+g(X, \varphi Z) \varphi Y-g(Y, \varphi Z) \varphi X+2 g(Y, \varphi Z) \varphi X \\
&+g(Y, \varphi X) \varphi Z-g(Z, \varphi X) \varphi Y \\
&+2 g(Z, \varphi X) \varphi Y+g(Z, \varphi Y) \varphi X-g(X, \varphi Y) \varphi Z .
\end{aligned}
$$

From (2.5) we get (3.4).

From (3.2) we have

$$
\begin{aligned}
\bar{T}(X, Y, Z, W)= & T(X, Y, Z, W)+g(Y, Z) \eta(X) \eta(W) \\
& -g(X, Z) \eta(Y) \eta(W)+g(X, W) \eta(Y) \eta(Z) \\
& -g(Y, W) \eta(X) \eta(Z)+2 g(X, \varphi Y) g(\varphi Z, W) \\
& +g(X, \varphi Z) g(\varphi Y, W)-g(Y, \varphi Z) g(\varphi X, W) .
\end{aligned}
$$

It is well known that

$$
\begin{gathered}
T(X, Y, Z, W)=-T(Y, X, Z, W), \quad T(X, Y, Z, W)=T(X, Y, W, Z), \\
T(X, Y, Z, W)=T(Z, W, X, Y) .
\end{gathered}
$$

By taking into account the previous equations we get (3.5), (3.6), and (3.7), respectively.

Let $E_{i}=\left\{e_{i}, \varphi e_{i}, \xi\right\}(i=1,2, \ldots, n)$ be a local orthonormal $\varphi$-basis of a para-Sasakian manifold $M$. Then the Ricci tensor $\bar{S}$ and the scalar curvature $\bar{\tau}$ of $M$ with respect to canonical paracontact connection $\bar{\nabla}$ are defined by

$$
\begin{aligned}
\bar{S}(X, Y)= & \sum_{i=1}^{n} g\left(\bar{R}\left(e_{i}, X\right) Y, e_{i}\right)-\sum_{i=1}^{n} g\left(\bar{R}\left(\varphi e_{i}, X\right) Y, \varphi e_{i}\right) \\
& +g(\bar{R}(\xi, X) Y, \xi)
\end{aligned}
$$


where $X, Y \in \Gamma(T M)$, and

$$
\bar{\tau}=\sum_{i=1}^{n} \bar{S}\left(e_{i}, e_{i}\right)-\sum_{i=1}^{n} \bar{S}\left(\varphi e_{i}, \varphi e_{i}\right)+\bar{S}(\xi, \xi)
$$

respectively.

Theorem 3.2. In a $(2 n+1)$-dimensional para-Sasakian manifold the Ricci tensor $\bar{S}$ and scalar curvature $\bar{\tau}$ of canonical paracontact connection $\bar{\nabla}$ are given by

$$
\begin{gathered}
\bar{S}(X, Y)=S(X, Y)-2 g(X, Y)+(2 n+2) \eta(X) \eta(Y), \\
\bar{\tau}=\tau-2 n,
\end{gathered}
$$

where $X, Y \in \Gamma(T M)$ and $S$ and $\tau$ denote the Ricci tensor and scalar curvature of Levi-Civita connection $\nabla$, respectively. Consequently, $\bar{S}$ is symmetric.

Proof. Using (3.2) and (3.9), we have, for any $X, Y \in \Gamma(T M)$,

$$
\begin{aligned}
\bar{S}(X, Y)= & \sum_{i=1}^{n}\left(\begin{array}{c}
g\left(R\left(e_{i}, X\right) Y, e_{i}\right)+g\left(e_{i}, e_{i}\right) \eta(X) \eta(Y) \\
+3 g\left(e_{i}, \varphi X\right) g\left(\varphi Y, e_{i}\right)
\end{array}\right) \\
& -\sum_{i=1}^{n}\left(\begin{array}{c}
g\left(R\left(\varphi e_{i}, X\right) Y, \varphi e_{i}\right)+g\left(\varphi e_{i}, \varphi e_{i}\right) \eta(X) \eta(Y) \\
+3 g\left(\varphi e_{i}, \varphi X\right) g\left(\varphi Y, \varphi e_{i}\right)
\end{array}\right) .
\end{aligned}
$$

Since the Ricci tensor of Levi-Civita connection $\nabla$ is given by

$$
S(X, Y)=\sum_{i=1}^{n} g\left(R\left(e_{i}, X\right) Y, e_{i}\right)-\sum_{i=1}^{n} g\left(R\left(\varphi e_{i}, X\right) Y, \varphi e_{i}\right)+g(R(\xi, X) Y, \xi)
$$

then (3.15) implies (3.13). Equation (3.14) follows from (3.13).

Also from (3.13), it is obvious that $\bar{S}$ is symmetric.

Corollary 3.3. If a para-Sasakian manifold is Ricci-flat with respect to canonical paracontact connection, then it is an $\eta$-Einstein manifold.

Lemma 3.4. Let $M$ be a para-Sasakian manifold with canonical paracontact connection $\bar{\nabla}$. Then

$$
\begin{gathered}
g(\bar{R}(X, Y) Z, \xi)=\eta(\bar{R}(X, Y) Z)=0 \\
\bar{R}(X, Y) \xi=\bar{R}(\xi, X) Y=\bar{R}(\xi, X) \xi=0 \\
\bar{S}(X, \xi)=0
\end{gathered}
$$

for all $X, Y, Z \in \Gamma(T M)$. 
International Journal of Mathematics and Mathematical Sciences

Proof. Calculation is straightforward by using (2.2), (2.6), (2.13), and (2.16) in (3.2).

\section{Conformal Curvature Tensor}

Let $M$ be a $(2 n+1)$-dimensional para-Sasakian manifold. The conformal curvature tensor of $M$ with respect to canonical paracontact connection $\bar{\nabla}$ is defined by

$$
\begin{aligned}
\bar{C}(X, Y) Z= & \bar{R}(X, Y) Z-\frac{1}{2 n-1}\left(\begin{array}{c}
\bar{S}(Y, Z) X-\bar{S}(X, Z) Y \\
+g(Y, Z) \bar{Q} X-g(X, Z) \bar{Q} Y
\end{array}\right) \\
& +\frac{\bar{\tau}}{2 n(2 n-1)}(g(Y, Z) X-g(X, Z) Y),
\end{aligned}
$$

where $X, Y, Z \in \Gamma(T M)$. By using (3.2), (3.13), and (3.14) in (4.1)

$$
\begin{aligned}
\bar{C}(X, Y) Z= & R(X, Y) Z+g(Y, Z) \eta(X) \xi-g(X, Z) \eta(Y) \xi \\
& +\eta(Y) \eta(Z) X-\eta(X) \eta(Z) Y+2 g(X, \varphi Y) \varphi Z \\
& +g(X, \varphi Z) \varphi Y-g(Y, \varphi Z) \varphi X \\
& -\frac{1}{2 n-1}\left(\begin{array}{c}
S(Y, Z) X-2 g(Y, Z) X \\
+(2 n+2) \eta(Y) \eta(Z) X \\
-S(X, Z) Y+2 g(X, Z) Y \\
-(2 n+2) \eta(X) \eta(Z) Y \\
+g(Y, Z) Q X-2 g(Y, Z) X \\
+(2 n+2) g(Y, Z) \eta(X) \xi \\
-g(X, Z) Q Y+2 g(X, Z) Y \\
-(2 n+2) g(X, Z) \eta(Y) \xi
\end{array}\right) \\
& +\frac{\tau-2 n}{2 n(2 n-1)}(g(Y, Z) X-g(X, Z) Y) .
\end{aligned}
$$

Since $R(X, Y) Z=-R(Y, X) Z$, then

$$
\bar{C}(X, Y) Z+\bar{C}(Y, X) Z=0
$$

Moreover, from the first Bianchi identity we get

$$
\bar{C}(X, Y) Z+\bar{C}(Y, Z) X+\bar{C}(Z, X) Y=0
$$

\section{5. $W_{2}$-Curvature Tensor}

In [15] Pokhariyal and Mishra have introduced a new tensor field, called $W_{2}$-curvature tensor field, in a Riemannian manifold and studied its properties. 
The $W_{2}$-curvature tensor is defined by

$$
W_{2}(X, Y, Z, V)=R(X, Y, Z, V)+\frac{1}{n-1}(g(X, Z) S(Y, V)-g(Y, Z) S(X, V))
$$

where $X, Y, Z, V \in \Gamma(T M)$ and $S$ is a Ricci tensor of type $(0,2)$. The $\bar{W}_{2}$-curvature tensor of a para-Sasakian manifold $M^{2 n+1}$ with respect to canonical paracontact connection $\bar{\nabla}$ is defined by

$$
\bar{W}_{2}(X, Y) Z=\bar{R}(X, Y) Z-\frac{1}{2 n}(g(Y, Z) \bar{Q} X-g(X, Z) \bar{Q} Y)
$$

From (3.2) and (3.13) we get

$$
\begin{aligned}
\bar{W}_{2}(X, Y) Z= & R(X, Y) Z+g(Y, Z) \eta(X) \xi-g(X, Z) \eta(Y) \xi \\
& +\eta(Y) \eta(Z) X-\eta(X) \eta(Z) Y+2 g(X, \varphi Y) \varphi Z \\
& +g(X, \varphi Z) \varphi Y-g(Y, \varphi Z) \varphi X \\
& -\frac{1}{2 n-1}\left(\begin{array}{c}
g(Y, Z) Q X-2 g(Y, Z) X \\
+(2 n+2) g(Y, Z) \eta(X) \xi \\
-g(X, Z) Q Y+2 g(X, Z) Y \\
-(2 n+2) g(X, Z) \eta(Y) \xi
\end{array}\right) .
\end{aligned}
$$

Since $R(X, Y) Z+R(Y, X) Z=0$, we have

$$
\bar{W}_{2}(X, Y) Z+\bar{W}_{2}(Y, X) Z=0
$$

Furthermore, by using (5.3) and the first Bianchi identity we obtain

$$
\bar{W}_{2}(X, Y) Z+\bar{W}_{2}(Y, Z) X+\bar{W}_{2}(Z, X) Y=0
$$

\section{Para-Sasakian Manifold with Canonical Paracontact Connection Satisfying $\bar{C}(\xi, X) \cdot \bar{R}=0$}

In this section we consider a para-Sasakian manifold $M^{2 n+1}$ satisfying the condition

$$
\bar{C}(\xi, X) \cdot \bar{R}=0,
$$


International Journal of Mathematics and Mathematical Sciences

with respect to canonical paracontact connection. From (2.20) we get

$$
\begin{aligned}
(\bar{C}(\xi, X) \cdot \bar{R})(U, V) W= & \bar{C}(\xi, X) \bar{R}(U, V) W-\bar{R}(\bar{C}(\xi, X) U, V) W \\
& -\bar{R}(U, \bar{C}(\xi, X) V) W-\bar{R}(U, V) \bar{C}(\xi, X) W \\
= & 0,
\end{aligned}
$$

for any $X, U, V, W \in \Gamma(T M)$. Now putting $U=\xi$ in (6.2), we have

$$
\begin{aligned}
& \bar{C}(\xi, X) \bar{R}(\xi, V) W-\bar{R}(\bar{C}(\xi, X) \xi, V) W-\bar{R}(\xi, \bar{C}(\xi, X) V) W-\bar{R}(\xi, V) \bar{C}(\xi, X) W \\
& \quad=0 .
\end{aligned}
$$

Taking inner product with $Y$ and using (3.18) in (6.3) we get

$$
g(\bar{R}(\bar{C}(\xi, X) \xi, V) W, Y)=0
$$

Using (4.2) in (6.4), we obtain

$$
\frac{1}{2 n-1} g(\bar{R}(W, Y) \bar{Q} X, V)-\frac{\bar{\tau}}{2 n(2 n-1)} g(\bar{R}(W, Y) X, V)=0
$$

Again using (3.2), (3.13), and (3.14) in (6.5) we obtain

$$
\begin{gathered}
\frac{1}{2 n-1}\left[\begin{array}{c}
g(R(W, Y) Q X, V)+g(Y, Q X) \eta(V) \eta(W) \\
-g(W, Q X) \eta(Y) \eta(V)+g(W, V) \eta(Q X) \eta(Y) \\
-g(Y, V) \eta(Q X) \eta(W)+2 g(W, \varphi Y) g(\varphi Q X, V) \\
+g(W, \varphi Q X) g(\varphi Y, V)-g(Y, \varphi Q X) g(\varphi W, V)
\end{array}\right] \\
-\frac{\tau-2 n}{2 n(2 n-1)}\left[\begin{array}{c}
g(R(W, Y) X, V)+g(Y, X) \eta(V) \eta(W) \\
-g(W, X) \eta(Y) \eta(V)+g(W, V) \eta(X) \eta(Y) \\
-g(Y, V) \eta(X) \eta(W)+2 g(W, \varphi Y) g(\varphi X, V) \\
+g(W, \varphi X) g(\varphi Y, V)-g(Y, \varphi X) g(\varphi W, V)
\end{array}\right]=0 .
\end{gathered}
$$

Let $\left\{e_{i}, \varphi e_{i}, \xi\right\}(i=1, \ldots, n)$ be an orthonormal basis of the tangent space at any point. Hence by suitable contracting of (6.6) we get

$$
\frac{1}{2 n-1}\left[\begin{array}{c}
S^{2}(X, Y)-4 S(X, Y)+4 g(X, Y) \\
-\left(4 n^{2}+8 n+4\right) \eta(X) \eta(Y)
\end{array}\right]-\frac{\tau-2 n}{2 n(2 n-1)}\left[\begin{array}{c}
S(X, Y)-2 g(X, Y) \\
+(2 n+2) \eta(X) \eta(Y)
\end{array}\right]=0
$$


Theorem 6.1. Let $M^{2 n+1}$ be a para-Sasakian manifold satisfying $\bar{C}(\xi, X) \cdot \bar{R}=0$ with respect to canonical paracontact connection $\bar{\nabla}$. Then

$$
\begin{aligned}
S(Q X, Y)= & \left(3+\frac{\tau}{2 n}\right) S(X, Y)-\left(2+\frac{\tau}{n}\right) g(X, Y) \\
& +\left(4 n^{2}+6 n+2+\frac{\tau(n+1)}{n}\right) \eta(X) \eta(Y)
\end{aligned}
$$

for any $X, Y \in \Gamma(T M)$.

\section{Para-Sasakian Manifold with Canonical Paracontact Connection}

Satisfying $\bar{W}_{2}(\xi, X) \cdot \bar{R}=0$ and $\bar{W}_{2}(\xi, X) \cdot \bar{W}_{2}=0$

In this section we consider a para-Sasakian manifold with canonical connection satisfying the condition

$$
\bar{W}_{2}(\xi, X) \cdot \bar{R}=0
$$

From (2.21), we get

$$
\begin{aligned}
\left(\bar{W}_{2}(\xi, X) \cdot \bar{R}\right)(U, V) W= & \bar{W}_{2}(\xi, X) \bar{R}(U, V) W-\bar{R}\left(\bar{W}_{2}(\xi, X) U, V\right) W \\
& -\bar{R}\left(U, \bar{W}_{2}(\xi, X) V\right) W-\bar{R}(U, V) \bar{W}_{2}(\xi, X) W \\
= & 0,
\end{aligned}
$$

where $X, U, V, W \in \Gamma(T M)$. Now if we put $U=\xi$ in (7.2), we have

$$
\begin{aligned}
\bar{W}_{2}(\xi, X) \bar{R}(\xi, V) W-\bar{R}\left(\bar{W}_{2}(\xi, X) \xi, V\right) W \\
\quad-\bar{R}\left(\xi, \bar{W}_{2}(\xi, X) V\right) W-\bar{R}(\xi, V) \bar{W}_{2}(\xi, X) W \\
\quad=0 .
\end{aligned}
$$

Taking inner product with $Y$ and from (3.18), we obtain 
International Journal of Mathematics and Mathematical Sciences

$$
g\left(\bar{R}\left(\bar{W}_{2}(\xi, X) \xi, V\right) W, Y\right)=0 .
$$

Using (5.3) in (7.4), we have

$$
\frac{1}{2 n} g(\bar{R}(W, Y) \bar{Q} X, V)=0
$$

Again using (3.2) and (3.13) in (7.5) we obtain

$$
\frac{1}{2 n}\left[\begin{array}{c}
g(R(W, Y) Q X, V)+g(Y, Q X) \eta(V) \eta(W) \\
-g(W, Q X) \eta(Y) \eta(V)+g(W, V) \eta(Q X) \eta(Y) \\
-g(Y, V) \eta(Q X) \eta(W)+2 g(W, \varphi Y) g(\varphi Q X, V) \\
+g(W, \varphi Q X) g(\varphi Y, V)-g(Y, \varphi Q X) g(\varphi W, V) \\
-2 g(R(W, Y) X, V)-2 g(Y, X) \eta(V) \eta(W) \\
+2 g(W, X) \eta(Y) \eta(V)-2 g(W, V) \eta(X) \eta(Y) \\
+2 g(Y, V) \eta(X) \eta(W)-4 g(W, \varphi Y) g(\varphi X, V) \\
-2 g(W, \varphi X) g(\varphi Y, V)+2 g(Y, \varphi X) g(\varphi W, V)
\end{array}\right]=0
$$

Let $\left\{e_{i}, \varphi e_{i}, \xi\right\}(i=1, \ldots, n)$ be an orthonormal basis of the tangent space at any point. So by a contraction of (7.6) with respect to $V$ and $W$ we get

$$
\frac{1}{2 n}\left[\begin{array}{c}
S^{2}(X, Y)-4 S(X, Y)+4 g(X, Y) \\
-\left(4 n^{2}+8 n+4\right) \eta(X) \eta(Y)
\end{array}\right]=0
$$

Theorem 7.1. Let $M^{2 n+1}$ be a para-Sasakian manifold satisfying $\bar{W}_{2}(\xi, X) \cdot \bar{R}=0$ with respect to canonical paracontact connection $\bar{\nabla}$. Then

$$
S(Q X, Y)=4 S(X, Y)-4 g(X, Y)+\left(4 n^{2}+8 n+4\right) \eta(X) \eta(Y)
$$

for any $X, Y \in \Gamma(T M)$.

Now let us consider a para-Sasakian manifold with canonical paracontact connection satisfying the condition

$$
\bar{W}_{2}(\xi, X) \cdot \bar{W}_{2}=0 .
$$

From (2.22), we get

$$
\begin{aligned}
\left(\bar{W}_{2}(\xi, X) \cdot \bar{W}_{2}\right)(U, V) Z= & \bar{W}_{2}(\xi, X) \bar{W}_{2}(U, V) Z-\bar{W}_{2}\left(\bar{W}_{2}(\xi, X) U, V\right) Z \\
& -\bar{W}_{2}\left(U, \bar{W}_{2}(\xi, X) V\right) Z-\bar{W}_{2}(U, V) \bar{W}_{2}(\xi, X) Z \\
= & 0 .
\end{aligned}
$$


Now $U=\xi$ in (7.10), we have

$$
\begin{aligned}
\bar{W}_{2}(\xi, X) \bar{W}_{2}(\xi, V) Z-\bar{W}_{2}\left(\bar{W}_{2}(\xi, X) \xi, V\right) Z \\
\quad-\bar{W}_{2}\left(\xi, \bar{W}_{2}(\xi, X) V\right) Z-\bar{W}_{2}(\xi, V) \bar{W}_{2}(\xi, X) Z \\
=0 .
\end{aligned}
$$

Taking inner product with $Y$ and from (3.18) and (3.19) we obtain

$$
\frac{1}{2 n} g\left(\bar{W}_{2}(\bar{Q} X, V) Z, Y\right)-\frac{1}{4 n^{2}}\left[g\left(\bar{Q}^{2} X, Y\right) \eta(V) \eta(Z)\right]=0
$$

Using (5.3) in (7.12), we have

$$
\frac{1}{2 n} g(\bar{R}(\bar{Q} X, V) Z, Y)-\frac{1}{4 n^{2}}\left[\begin{array}{c}
g\left(\bar{Q}^{2} X, Y\right) g(V, Z)-g(\bar{Q} X, Z) g(\bar{Q} V, Y) \\
+g\left(\bar{Q}^{2} X, Y\right) \eta(V) \eta(Z)
\end{array}\right]=0
$$

Let $\left\{e_{i}, \varphi e_{i}, \xi\right\}(i=1, \ldots, n)$ be an orthonormal basis of the tangent space at any point. Hence by suitable contracting (7.13) we get

$$
\frac{1}{2 n}\left[\begin{array}{c}
S^{2}(X, Y)-4 S(X, Y)+4 g(X, Y) \\
-\left(4 n^{2}+8 n+4\right) \eta(X) \eta(Y)
\end{array}\right]-\frac{1}{4 n^{2}}\left[\begin{array}{c}
S^{2}(X, Y)-4 S(X, Y)+4 g(X, Y) \\
-\left(4 n^{2}+8 n+4\right) \eta(X) \eta(Y)
\end{array}\right]=0
$$

Theorem 7.2. Let $M^{2 n+1}$ be a para-Sasakian manifold satisfying $\bar{W}_{2}(\xi, X) \cdot \bar{W}_{2}=0$ with respect to canonical paracontact connection $\bar{\nabla}$. Then

$$
S(Q X, Y)=4 S(X, Y)-4 g(X, Y)+\left(4 n^{2}+8 n+4\right) \eta(X) \eta(Y)
$$

for any $X, Y \in \Gamma(T M)$.

\section{Concircularly Flat Para-Sasakian Manifold}

The concircular curvature tensor of a para-Sasakian manifold $M^{2 n+1}$ with respect to canonical paracontact connection $\bar{\nabla}$ is defined by

$$
\bar{Z}(X, Y) W=\bar{R}(X, Y) W-\frac{\bar{\tau}}{2 n(2 n-1)}(g(Y, W) X-g(X, W) Y)
$$

for any $X, Y, W \in \Gamma(T M)$. 
By using (3.2) and (3.14) we obtain from (8.1)

$$
\begin{aligned}
\bar{Z}(X, Y) W= & R(X, Y) W+g(Y, W) \eta(X) \xi-g(X, W) \eta(Y) \xi \\
& +\eta(Y) \eta(W) X-\eta(X) \eta(W) Y+2 g(X, \varphi Y) \varphi W \\
& +g(X, \varphi W) \varphi Y-g(Y, \varphi W) \varphi X \\
& -\frac{\tau-2 n}{2 n(2 n-1)}(g(Y, W) X-g(X, W) Y) .
\end{aligned}
$$

If $M$ is a concircularly flat para-Sasakian manifold with respect to canonical paracontact connection, then we have

$$
g(\bar{Z}(X, Y) W, \varphi V)=0
$$

Hence using (2.2) in (8.3) we get

$$
\begin{aligned}
g(R(X, Y) W, \varphi V)+g(X, \varphi V) \eta(Y) \eta(W) \\
\quad-g(Y, \varphi V) \eta(X) \eta(W)+2 g(X, \varphi Y) g(\varphi W, \varphi V) \\
\quad+g(X, \varphi W) g(\varphi Y, \varphi V)-g(Y, \varphi W) g(\varphi X, \varphi V) \\
\quad-\frac{\tau-2 n}{2 n(2 n+1)}(g(Y, W) g(X, \varphi V)-g(X, W) g(Y, \varphi V)) \\
=0 .
\end{aligned}
$$

Putting $Y=W=\xi$ in (8.4) and using (2.2) and (2.14) we have

$$
\frac{\tau-2 n}{2 n(2 n+1)} g(X, \varphi V)=0
$$

From (8.5) we obtain

$$
\tau=2 n \text {. }
$$

Hence we have the following.

Theorem 8.1. If a para-Sasakian manifold $M$ is concircularly flat with respect to canonical paracontact connection, then it is of constant scalar curvature.

\section{Pseudo-projectively Flat Para-Sasakian Manifold}

In 2002, Prasad [16] defined and studied a tensor field $\bar{P}$ on a Riemannian manifold of dimension $n$, which includes projective curvature tensor $P$. This tensor field $\bar{P}$ is known as pseudo-projective curvature tensor. 
In this section, we study pseudo-projective curvature tensor with respect to canonical paracontact connection $\bar{\nabla}$ in a para-Sasakian manifold and we denote this curvature tensor with $\bar{P} \bar{P}$.

Pseudo-projective curvature tensor $\bar{P} \bar{P}$ of a para-Sasakian manifold $M^{2 n+1}$ with respect to canonical paracontact connection $\bar{\nabla}$ is defined by

$$
\begin{aligned}
\bar{P} \bar{P}(X, Y) W= & a R(X, Y) W+b(S(Y, W) X-S(X, W) Y) \\
& -\frac{\bar{\tau}}{(2 n+1)}\left(\frac{a}{2 n}+b\right)(g(Y, W) X-g(X, W) Y),
\end{aligned}
$$

where $X, Y, W \in \Gamma(T M)$ and $a$ and $b$ are constants such that $a, b \neq 0$.

If $a=1$ and $b=1 /(2 n+2)$, then (9.1) takes the form

$$
\begin{aligned}
\bar{P} \bar{P}(X, Y) W= & \bar{R}(X, Y) W+\frac{1}{2 n+2}(\bar{S}(Y, W) X-\bar{S}(X, W) Y) \\
& -\frac{\bar{\tau}}{(2 n+2) n}(g(Y, W) X-g(X, W) Y) .
\end{aligned}
$$

Using (3.2), (3.13) and (3.14) in (9.2), we get

$$
\begin{aligned}
\bar{P} \bar{P}(X, Y) W= & R(X, Y) W+g(Y, W) \eta(X) \xi-g(X, W) \eta(Y) \xi \\
& +\eta(Y) \eta(W) X-\eta(X) \eta(W) Y+2 g(X, \varphi Y) \varphi W \\
& +g(X, \varphi W) \varphi Y-g(Y, \varphi W) \varphi X \\
& +\frac{1}{2 n+2}\left(\begin{array}{c}
S(Y, W) X-2 g(Y, W) X \\
+(2 n+2) \eta(Y) \eta(W) X \\
-S(X, W) Y+2 g(X, W) Y \\
-(2 n+2) \eta(X) \eta(W) Y
\end{array}\right) \\
& -\frac{\tau-2 n}{(2 n+1)}(g(Y, W) X-g(X, W) Y) .
\end{aligned}
$$

If $M$ is a pseudo-projectively flat para-Sasakian manifold with respect to canonical paracontact connection, then

$$
g(\bar{P} \bar{P}(X, Y) W, \varphi U)=0
$$


for any $U \in \Gamma(T M)$. Hence using (2.2) in (9.4) we get

$$
\begin{aligned}
& g(R(X, Y) W, \varphi U)+g(X, \varphi U) \eta(Y) \eta(W) \\
& -g(Y, \varphi U) \eta(X) \eta(W)+2 g(X, \varphi Y) g(\varphi W, \varphi U) \\
& +g(X, \varphi W) g(\varphi Y, \varphi U)-g(Y, \varphi W) g(\varphi X, \varphi U) \\
& +\frac{1}{2 n+2}\left(\begin{array}{c}
S(Y, W) g(X, \varphi U)-2 g(Y, W) g(X, \varphi U) \\
+(2 n+2) g(X, \varphi U) \eta(Y) \eta(W) \\
-S(X, W) g(Y, \varphi U)+2 g(X, W) g(Y, \varphi U) \\
-(2 n+2) g(Y, \varphi U) \eta(X) \eta(W)
\end{array}\right) \\
& \quad-\frac{\tau-8 n}{(2 n+2) n}(g(Y, W) g(X, \varphi U)-g(X, W) g(Y, \varphi U)) \\
& =0 .
\end{aligned}
$$

Now putting $Y=W=\xi$ in (9.5) and using (2.2), (2.14), and (2.16) we have

$$
\frac{\tau-2 n}{(2 n+2) n} g(X, \varphi U)=0
$$

From (9.6) we get

$$
\tau=2 n \text {. }
$$

Hence we have the following.

Theorem 9.1. If a para-Sasakian manifold is pseudo-projectively flat with respect to canonical paracontact connection, then its scalar curvature is constant.

\section{Para-Sasakian Manifold with Canonical Paracontact Connection Satisfying $\bar{Z}(\xi, X) \cdot \bar{R}=0$ and $\bar{Z}(\xi, X) \cdot \bar{S}=0$}

In this section we firstly consider a para-Sasakian manifold $M$ satisfying

$$
\bar{Z}(\xi, X) \cdot \bar{R}=0,
$$

for any $X \in \Gamma(T M)$, with respect to canonical paracontact connection.

From (2.23), we have

$$
\begin{aligned}
(\bar{Z}(\xi, X) \cdot \bar{R})(U, V) W= & \bar{Z}(\xi, X) \bar{R}(U, V) W-\bar{R}(\bar{Z}(\xi, X) U, V) W \\
& -\bar{R}(U, \bar{Z}(\xi, X) V) W-\bar{R}(U, V) \bar{Z}(\xi, X) W \\
= & 0,
\end{aligned}
$$


for all $X, U, V, W \in \Gamma(T M)$. Now by putting $U=\xi$ in (10.2), we get

$$
\begin{aligned}
\bar{Z}(\xi, X) \bar{R}(\xi, V) W-\bar{R}(\bar{Z}(\xi, X) \xi, V) W \\
-\bar{R}(\xi, \bar{Z}(\xi, X) V) W-\bar{R}(\xi, V) \bar{Z}(\xi, X) W=0 .
\end{aligned}
$$

Using (3.18) in (10.3) we obtain

$$
\bar{R}(\bar{Z}(\xi, X) \xi, V) W=0
$$

Taking inner product with $Y$ and from (8.2), we have

$$
\frac{\tau-2 n}{2 n(2 n-1)} g(\bar{R}(X, V) W, Y)=0
$$

Again using (3.2) in (10.5) we get

$$
\frac{\tau-2 n}{2 n(2 n-1)}\left[\begin{array}{c}
g(R(X, V) W, Y)+g(V, W) \eta(X) \eta(Y) \\
-g(X, W) \eta(Y) \eta(V)+g(X, Y) \eta(V) \eta(W) \\
-g(Y, V) \eta(X) \eta(W)+2 g(X, \varphi V) g(\varphi W, Y) \\
+g(X, \varphi W) g(Y, \varphi V)-g(V, \varphi W) g(\varphi X, Y)
\end{array}\right]=0
$$

Let $\left\{e_{i}, \varphi e_{i}, \xi\right\}(i=1, \ldots, n)$ be an orthonormal basis of the tangent space at any point. So a contraction of (10.6) with respect to $X$ and $Y$ gives

$$
\frac{\tau-2 n}{2 n(2 n-1)}\left[\begin{array}{l}
S(V, W)-2 g(V, W) \\
+(2 n+2) \eta(V) \eta(W)
\end{array}\right]=0
$$

Therefore we have the following.

Theorem 10.1. Let $M^{2 n+1}$ be a para-Sasakian manifold satisfying the condition $\bar{Z}(\xi, X) \cdot \bar{R}=0$, for any $X \in \Gamma(T M)$. Then either

(i) $\tau=2 n$, that is, the scalar curvature is constant,

or

(ii) $M$ is an $\eta$-Einstein manifold with equation

$$
S(V, W)=2 g(V, W)-(2 n+2) \eta(V) \eta(W),
$$

for all $V, W \in \Gamma(T M)$.

Now, let us consider a para-Sasakian manifold $M$ satisfying

$$
\bar{Z}(\xi, X) \cdot \bar{S}=0,
$$


where $X \in \Gamma(T M)$ and $\bar{S}$ is the Ricci tensor of $M$ with respect to canonical paracontact connection.

From (10.9), for any $X, U, V \in \Gamma(T M)$, we obtain

$$
\bar{S}(\bar{Z}(\xi, X) U, V)+\bar{S}(U, \bar{Z}(\xi, X) V)=0,
$$

for all $X, U, V \in \Gamma(T M)$. Using (3.18) and (3.19) with (8.2) in (10.10) we get

$$
A[\bar{S}(X, V) \eta(U)+\bar{S}(X, U) \eta(V)]=0,
$$

where $A=\bar{\tau} / 2 n(2 n-1)$.

Using (3.13) and (3.14) in (10.11), we get

$$
\frac{\tau-2 n}{2 n(2 n-1)}\left[\begin{array}{c}
S(X, V) \eta(U)-2 g(X, V) \eta(U) \\
+(2 n+2) \eta(X) \eta(V) \eta(U) \\
-S(X, U) \eta(V)+2 g(X, U) \eta(V) \\
-(2 n+2) \eta(X) \eta(V) \eta(U)
\end{array}\right]=0 .
$$

Let $\left\{e_{i}, \varphi e_{i}, \xi\right\}(i=1, \ldots, n)$ be an orthonormal basis of the tangent space at any point. By a contraction of (10.12) with respect to $X$ and $U$ we obtain

$$
\frac{\tau-2 n}{2 n(2 n-1)}[(\tau-2 n) \eta(V)]=0
$$

This gives $\tau=2 n$ which implies that manifold is of constant scalar curvature.

Theorem 10.2. If the condition $\bar{Z}(\xi, X) \cdot \bar{S}=0$, for all $X \in \Gamma(T M)$, holds on a para-Sasakian manifold, then its scalar curvature is constant.

\section{Para-Sasakian Manifold with Canonical Paracontact Connection Satisfying $\bar{P} \bar{P}(\xi, X) \cdot \bar{R}=0$ and $\bar{P} \bar{P}(\xi, X) \cdot \bar{S}=0$}

In this section we consider a para-Sasakian manifold $M^{2 n+1}$ with canonical paracontact connection satisfying the condition

$$
\bar{P} \bar{P}(\xi, X) \cdot \bar{R}=0
$$

for all $X \in \Gamma(T M)$.

From (2.24), we get

$$
\begin{aligned}
(\bar{P} \bar{P}(\xi, X) \cdot \bar{R})(U, V) W= & \bar{P} \bar{P}(\xi, X) \bar{R}(U, V) W-\bar{R}(\bar{P} \bar{P}(\xi, X) U, V) W \\
& -\bar{R}(U, \bar{P} \bar{P}(\xi, X) V) W-\bar{R}(U, V) \bar{P} \bar{P}(\xi, X) W \\
= & 0,
\end{aligned}
$$


where $X, U, V, W \in \Gamma(T M)$. Now we take $U=\xi$ in (11.2), then we have

$$
\begin{aligned}
\bar{P} \bar{P}(\xi, X) \bar{R}(\xi, V) W-\bar{R}(\bar{P} \bar{P}(\xi, X) \xi, V) W \\
-\bar{R}(\xi, \bar{P} \bar{P}(\xi, X) V) W-\bar{R}(\xi, V) \bar{P} \bar{P}(\xi, X) W=0 .
\end{aligned}
$$

Taking inner product with $Y \in \Gamma(T M)$ and by using (3.18) and (9.3) we obtain

$$
\frac{\tau-2 n}{(2 n+2) n} g(\bar{R}(X, V) W, Y)=0
$$

Again using (3.2) in (11.4), we have

$$
\frac{\tau-2 n}{(2 n+2) n}\left[\begin{array}{c}
g(R(X, V) W, Y)+g(V, W) \eta(X) \eta(Y) \\
-g(X, W) \eta(Y) \eta(V)+g(X, Y) \eta(V) \eta(W) \\
-g(Y, V) \eta(X) \eta(W)+2 g(X, \varphi V) g(\varphi W, Y) \\
+g(X, \varphi W) g(Y, \varphi V)-g(V, \varphi W) g(\varphi X, Y)
\end{array}\right]=0
$$

Let $\left\{e_{i}, \varphi e_{i}, \xi\right\}(i=1, \ldots, n)$ be an orthonormal basis of the tangent space at any point. Hence by suitable contracting of (11.5) we get

$$
\frac{\tau-2 n}{2 n(2 n-1)}\left[\begin{array}{l}
S(V, W)-2 g(V, W) \\
+(2 n+2) \eta(V) \eta(W)
\end{array}\right]=0
$$

Therefore we have the following.

Theorem 11.1. Let $M^{2 n+1}$ be a para-Sasakian manifold. If $\bar{P} \bar{P}(\xi, X) \cdot \bar{R}=0$ holds on $M$, then either

(i) scalar curvature is constant with equation $\tau=2 n$

or

(ii) $M$ is an $\eta$-Einstein manifold with equation

$$
S(V, W)=2 g(V, W)-(2 n+2) \eta(V) \eta(W),
$$

for all $V, W \in \Gamma(T M)$.

Now let us consider a para-Sasakian manifold satisfying

$$
\bar{P} \bar{P}(\xi, X) \cdot \bar{S}=0
$$

where $X \in \Gamma(T M)$ and $\bar{S}$ is the Ricci tensor of $M$ with respect to canonical paracontact connection. 
International Journal of Mathematics and Mathematical Sciences

From (11.8) we obtain

$$
\bar{S}(\bar{P} \bar{P}(\xi, X) Y, Z)+\bar{S}(Y, \bar{P} \bar{P}(\xi, X) Z)=0
$$

Using (3.18) and (3.19) with (9.3) in (11.9) we have

$$
B[\bar{S}(X, Z) \eta(Y)+\bar{S}(X, Y) \eta(Z)]=0,
$$

where $B=\bar{\tau} /(2 n+2) n$.

Using (3.13) and (3.14) in (11.10) we get

$$
\frac{\tau-2 n}{(2 n+2) n}\left[\begin{array}{c}
S(X, Z) \eta(Y)-2 g(X, Z) \eta(Y) \\
+(2 n+2) \eta(X) \eta(Y) \eta(Z) \\
-S(X, Y) \eta(Z)+2 g(X, Y) \eta(Z) \\
-(2 n+2) \eta(X) \eta(Y) \eta(Z)
\end{array}\right]=0
$$

Let $\left\{e_{i}, \varphi e_{i}, \xi\right\}(i=1, \ldots, n)$ be an orthonormal basis of the tangent space at any point. So a contraction of (11.11) with respect to $X$ and $Y$ gives

$$
\frac{\tau-2 n}{(2 n+2) n}[(\tau-2 n) \eta(Z)]=0,
$$

which implies that $\tau=2 n$.

So we have the following.

Theorem 11.2. If the condition $\bar{P} \bar{P}(\xi, X) \cdot \bar{S}=0$, for all $X \in \Gamma(T M)$, holds on a para-Sasakian manifold, then the scalar curvature of the manifold is constant.

\section{Para-Sasakian Manifold with Canonical Paracontact Connection Satisfying $\bar{P}(\xi, X) \cdot \bar{P} \bar{P}=0$}

The projective curvature tensor $\bar{P}$ of a para-Sasakian manifold $M^{2 n+1}$ with respect to canonical paracontact connection $\bar{\nabla}$ is defined by

$$
\bar{P}(X, Y) Z=\bar{R}(X, Y) Z-\frac{1}{2 n}(\bar{S}(Y, Z) X-\bar{S}(X, Z) Y),
$$


where $X, Y, Z \in \Gamma(T M)$. By using (3.2) and (3.13), from (12.1) we obtain

$$
\begin{aligned}
\bar{P}(X, Y) Z= & R(X, Y) Z+g(Y, Z) \eta(X) \xi-g(X, Z) \eta(Y) \xi \\
& +\eta(Y) \eta(Z) X-\eta(X) \eta(Z) Y+2 g(X, \varphi Y) \varphi Z \\
& +g(X, \varphi Z) \varphi Y-g(Y, \varphi Z) \varphi X \\
& -\frac{1}{2 n}\left(\begin{array}{c}
S(Y, Z) X-2 g(Y, Z) X \\
+(2 n+2) \eta(Y) \eta(Z) X \\
-S(X, Z) Y+2 g(X, Z) Y \\
-(2 n+2) \eta(X) \eta(Z) Y
\end{array}\right) .
\end{aligned}
$$

Let us consider the equation

$$
\bar{P}(\xi, X) \cdot \bar{P} \bar{P}=0
$$

holds on a para-Sasakian manifold. From (2.25), we get

$$
\begin{aligned}
(\bar{P}(\xi, X) \cdot \bar{P} \bar{P})(U, V) W= & \bar{P}(\xi, X) \bar{P} \bar{P}(U, V) W-\bar{P} \bar{P}(\bar{P}(\xi, X) U, V) W \\
& -\bar{P} \bar{P}(U, \bar{P}(\xi, X) V) W-\bar{P} \bar{P}(U, V) \bar{P}(\xi, X) W \\
= & 0,
\end{aligned}
$$

for all $X, U, V, W \in \Gamma(T M)$. Using (12.2) with (3.18) and (3.19) we obtain

$$
\frac{1}{2 n}\left[\begin{array}{l}
\bar{S}(X, \bar{P} \bar{P}(U, V) W) \xi-\bar{S}(X, U) \bar{P} \bar{P}(\xi, V) W \\
-\bar{S}(X, V) \bar{P} \bar{P}(U, \xi) W-\bar{S}(X, W) \bar{P} \bar{P}(U, V) \xi
\end{array}\right]=0 .
$$

Taking inner product with $Y$ and put $U=\xi$, by using (3.19) we get

$$
\frac{1}{2 n}[\bar{S}(X, \bar{P} \bar{P}(\xi, V) W) \eta(Y)-\bar{S}(X, W) g(\bar{P} \bar{P}(\xi, V) \xi, Y)]=0
$$

Using (9.3) in (12.6), we obtain

$$
C\left[\begin{array}{c}
\bar{S}(X, V) \eta(Y) \eta(W)+\bar{S}(X, W) \eta(Y) \eta(V) \\
-\bar{S}(X, W) g(Y, V)
\end{array}\right]=0
$$

where $C=\bar{\tau} /(2 n+2) 2 n^{2}$. 
From the last equation, with (3.13) we get

$$
C\left[\begin{array}{c}
S(X, V) \eta(Y) \eta(W)-2 g(X, V) \eta(Y) \eta(W) \\
+(2 n+2) \eta(X) \eta(Y) \eta(V) \eta(W) \\
+S(X, W) \eta(Y) \eta(V)-2 g(X, W) \eta(Y) \eta(V) \\
+(2 n+2) \eta(X) \eta(Y) \eta(V) \eta(W) \\
-S(X, W) g(Y, V)+2 g(X, W) g(Y, V) \\
-(2 n+2) \eta(X) \eta(Y) \eta(V) \eta(W)
\end{array}\right]=0
$$

Hence by suitable contracting of (12.8), we obtain

$$
\frac{\tau-2 n}{(2 n+2) 2 n^{2}}\left[\begin{array}{l}
S(V, W)-2 g(V, W) \\
+(2 n+2) \eta(V) \eta(W)
\end{array}\right]=0 .
$$

Hence we have the following.

Theorem 12.1. Let $M$ be a $(2 n+1)$-dimensional para-Sasakian manifold. If the condition $\bar{P}(\xi, X)$. $\bar{P} \bar{P}=0$ holds on $M$, then either

(i) scalar curvature is constant with equation $\tau=2 n$

or

(ii) $M$ is an $\eta$-Einstein manifold with equation

$$
S(V, W)=2 g(V, W)-(2 n+2) \eta(V) \eta(W),
$$

for all $V, W \in \Gamma(T M)$.

\section{Acknowledgments}

This paper was supported by Adıyaman University, under Scientific Research Project no. FEFBAP/2012-005. The authors thank the referee for useful suggestions and remarks for the revised version.

\section{References}

[1] I. Sato, "On a structure similar to the almost contact structure I.," Tensor N. S., vol. 30, pp. 219-224, 1976.

[2] S. Sasaki, "On differentiable manifolds with certain structures which are closely related to almost contact structure I," The Tohoku Mathematical Journal, vol. 12, no. 2, pp. 459-476, 1960.

[3] D. Blair, Contact Manifolds in Riemannian Geometry, vol. 509 of Lectures Notes in Mathematics, Springer, Berlin, Germany, 1976.

[4] T. Takahashi, "Sasakian manifold with pseudo-Riemannian metric," Tohoku Mathematical Journal, vol. 21, no. 2, pp. 644-653, 1969.

[5] K. Matsumoto, "On Lorentzian paracontact manifolds," Bulletin of the Yamagata University-Natural Science, vol. 12, no. 2, pp. 151-156, 1989.

[6] M. M. Tripathi, E. Kılıç, S. Y. Perktaş, and S. Keleş, "Indefinite almost paracontact metric manifolds," International Journal of Mathematics and Mathematical Sciences, vol. 2010, Article ID 846195, 19 pages, 2010. 
[7] S. Kaneyuki and M. Konzai, "Paracomplex structure and affine symmetric spaces," Tokyo Journal of Mathematics, vol. 8, pp. 301-318, 1985.

[8] S. Zamkovoy, "Canonical connections on paracontact manifolds," Annals of Global Analysis and Geometry, vol. 36, no. 1, pp. 37-60, 2009.

[9] S. Tanno, "Variational problems on contact Riemannian manifolds," Transactions of the American Mathematical Society, vol. 314, no. 1, pp. 349-379, 1989.

[10] N. Tanaka, "On non-degenerate real hypersurfaces, graded Lie algebras and Cartan connection," Japanese Journal of Mathematics, vol. 2, pp. 131-190, 1976.

[11] S. M. Webster, "Pseudo-Hermitian structures on a real hypersurfaces," Journal of Differential Geometry, vol. 13, pp. 25-41, 1979.

[12] P. Libermann, "Sur le probleme d'equivalence de certaines structures infinitesimales," Annali di Matematica Pura ed Applicata, vol. 36, pp. 27-120, 1954.

[13] K. Yano, "Affine connexions in almost product spaces," Kodai Mathematical Seminar Reports, vol. 11, pp. 1-24, 1959.

[14] S. Kaneyuki and F. L. Willams, "Almost paracontact and parahodge structure on manifolds," Nagoya Mathematics Journal, vol. 99, pp. 173-187, 1985.

[15] G. P. Pokhariyal and R. S. Mishra, "The curvature tensor and their relativistic significance," Yokohama Mathematical Journal, vol. 18, pp. 105-108, 1970.

[16] B. Prasad, "A Pseudo-Projective curvature tensor on a Riemannian manifold," Bulletin of Calcutta Mathematical Society, vol. 94, no. 3, pp. 163-166, 2002. 


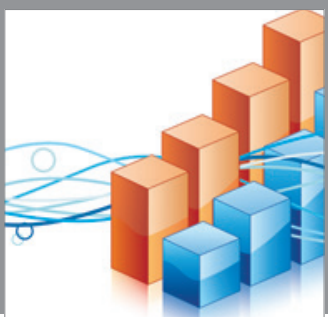

Advances in

Operations Research

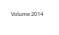

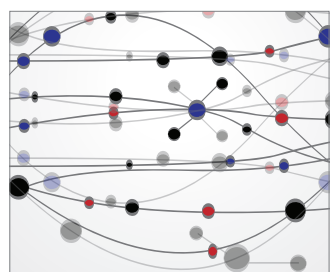

\section{The Scientific} World Journal
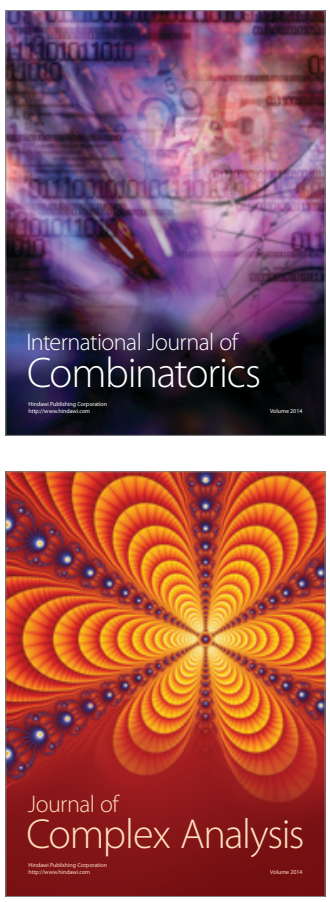

International Journal of

Mathematics and

Mathematical

Sciences
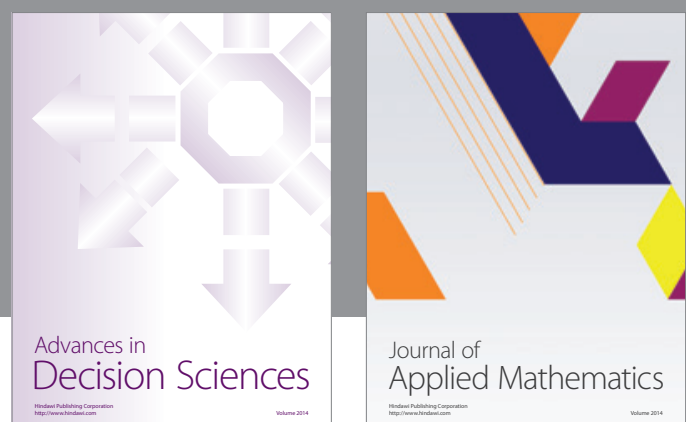

Journal of

Applied Mathematics
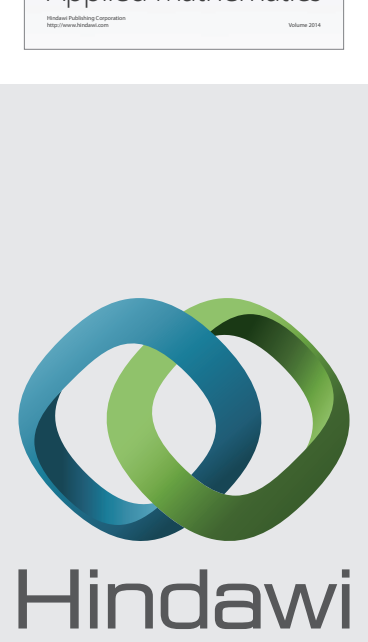

Submit your manuscripts at http://www.hindawi.com
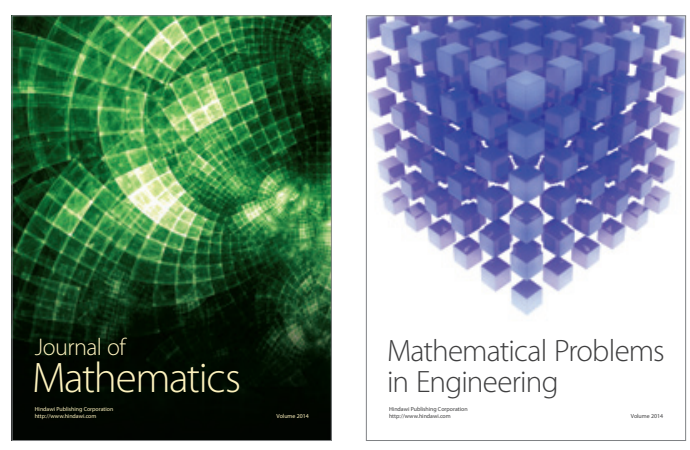

Mathematical Problems in Engineering
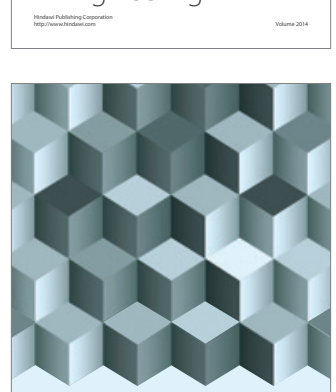

Journal of

Function Spaces
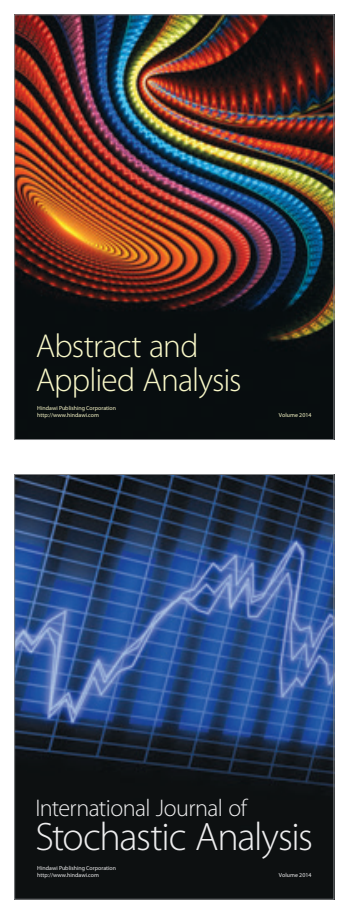

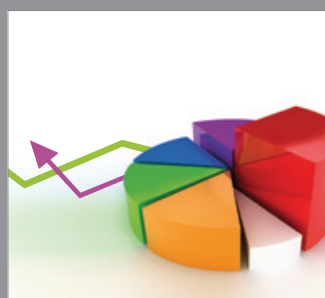

ournal of

Probability and Statistics

Promensencen
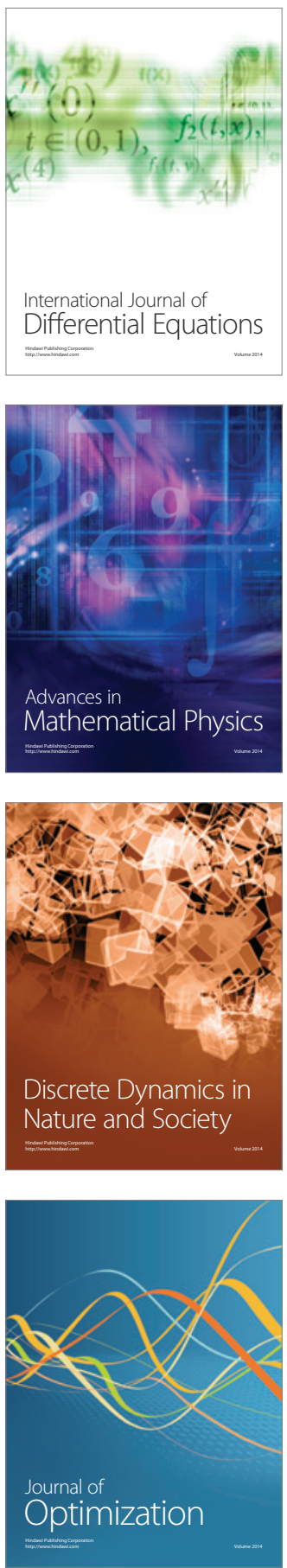